\title{
La « formation-symptôme » intervention et changement dans les organisations
}

Pierre de Saint-Georges

\section{OpenEdition}

\section{Journals}

Édition électronique

URL : http://journals.openedition.org/communicationorganisation/2363

DOI : 10.4000/communicationorganisation.2363

ISSN : 1775-3546

Éditeur

Presses universitaires de Bordeaux

Édition imprimée

Date de publication : 1 mai 2000

ISSN : 1168-5549

Référence électronique

Pierre de Saint-Georges, « La « formation-symptôme » intervention et changement dans les organisations », Communication et organisation [En ligne], 17 | 2000, mis en ligne le 19 décembre 2012, consulté le 10 décembre 2020. URL : http://journals.openedition.org/communicationorganisation/ 2363 ; DOI : https://doi.org/10.4000/communicationorganisation.2363

Ce document a été généré automatiquement le 10 décembre 2020.

(c) Presses universitaires de Bordeaux 


\title{
La « formation-symptôme » intervention et changement dans les organisations
}

\author{
Pierre de Saint-Georges
}

1 Dans le vaste champ du changement organisationnel, nous voudrions nous intéresser plus particulièrement ici aux changements qui concernent le management des personnes. Caricaturalement, le problème est vieux comme l'organisation de l'action humaine: comment décomposer un travail complexe entre plusieurs acteurs ou groupes d'acteurs et puis comment coordonner ces acteurs pour que l'opération aboutisse au but escompté, le tout avec un maximum d'efficacité (rapport buts-résultats) et d'efficience (rapport moyens-résultats). Du point de vue des personnes, il s'agit en quelque sorte d'être à la fois performants et satisfaits. Ce n'est pas faux, quel que soit le niveau hiérarchique auquel on se place.

2 Mais la vie des entreprises, des administrations ou des associations montre certains décalages entre la réalité de tous les jours et ces idéaux de performance et de satisfaction. Le rapport de coopération conflictuelle, en quelque sorte positivement constitutif de l'organisation, fluctue. Parfois il en arrive à se détériorer sous la pression de facteurs internes et/ou externes très divers, momentanés ou plus durables (du caractère des personnes en présence aux pressions de l'environnement, par exemple).

3 De ce point de vue, c'est la dynamique même de l'organisation qui réclame donc déjà une véritable gestion quotidienne du changement. Parfois elle le réclamera avec une telle acuité que cela nécessitera une "intervention », c'est-à-dire une démarche délibérée et méthodique d'induction de changement. Les projets de «culture d'entreprise» par exemple, ne sont souvent pas autre chose.

4 Sur cette toile de fond, nous allons proposer, dans un premier temps, une série de repères destinés à clarifier la notion d'intervention. Il nous semble à l'expérience que ces repères peuvent être utiles à la fois aux directions d'organisation qui ont à réfléchir aux méthodes d'induction du changement, aux intervenants spécialisés pour clarifier avec les organisations et pour eux-mêmes les stratégies de travail les plus indiquées ainsi qu'au 
chercheur pour approfondir cette problématique du changement voulu, mais pas toujours obtenu. Nous présenterons ensuite deux exemples pour discuter plus spécifiquement la place de la formation dans ce contexte et comprendre pourquoi certains projets de formation (portant sur le domaine que nous venons de cerner) sont parfois le symptôme d'une demande d'intervention qui paradoxalement devrait porter sur le changement que la formation elle-même croyait apporter.

Notre thèse est que la formation en tant que processus construit visant à un changement organisationnel est une modalité particulière d'intervention psychosociologique qui doit être pilotée comme telle dès le départ pour réussir, tant de la part de l'organisation que d'éventuels intervenants extérieurs à elle.

\section{La notion d'intervention psychosociologique}

6 Pour cerner et discuter la notion d'intervention psychosociologique dans sa dynamique et sa complexité, il nous semble fécond d'y relever l'action complémentaire de deux axes de significations: d'une part, la notion d'intervention implique nécessairement un positionnement des acteurs par rapport à un changement à opérer dans l'organisation ; c'est ce que nous appellerons le mode d'intervention. D'autre part, toute intervention suppose en même temps et indissociablement une relation entre le «système intervenant » (qu'il soit interne ou externe à l'organisation) et le « système client « (par hypothèse, l'organisation ou une partie de celle-ci). C'est ce que nous appellerons le type d'implication.

\section{L'axe du changement : le mode d'intervention}

7 On peut identifier plus précisément deux grandes options de travail par rapport à ce que nous venons d'appeler le mode d'intervention. Dans certains cas, l'intervention a pour mission spécifique de résoudre directement un problème au sein de l'organisation. Sa caractéristique principale dans cette hypothèse est de devoir induire un changement. Mais dans d'autres cas, l'intervention n'a pas nécessairement pour but de produire directement un changement ou de résoudre par elle-même un problème. La mission qui lui est assignée apparaît plutôt comme un traitement rigoureux d'informations prélevées méthodiquement dans l'organisation afin de nourrir la prise de décision par les instances qui en ont la responsabilité. L'intervention agit ici sur un mode investigatif. Elle explore la situation et propose éventuellement des pistes concrètes de décision et d'action, mais elle n'induit pas comme telle le changement : elle fonctionne comme une aide à la décision de changement.

\section{L'axe de l'interdépendance : le type d'implication}

8 Le second axe, le type d'implication, est défini quant à lui par la relation établie entre l'organisation et l'intervenant. Cet axe traduit donc le degré d'indépendance (ou d'interdépendance) entre les parties (système intervenant, interne ou non, et système client) concrétisé par le mandat d'intervention.

9 Le type le plus distancié laisse une complète latitude à l'intervenant sur tous les paramètres de son travail d'intervention : agenda général, définition du problème, choix des hypothèses, délimitation du champ d'investigation, méthodes à mettre en œuvre pour 
la collecte et le traitement des données, diffusion des résultats. Au fond, poussé à bout, cet axe conduit à une extériorité complète - bien que négociée - du système intervenant par rapport au système client.

10 Sur le mode impliqué, par contre, le mandat d'intervention conduit contractuellement à une relation plus ou moins étroite entre les parties au sein du système d'intervention (constitué des sous-systèmes intervenants et clients). Dans cette hypothèse, la gestion de la dynamique des comportements et des interactions fait partie intégrante du processus d'intervention et de la conduite du changement. Poussé à bout, cet axe conduirait, au contraire du précédent, à l'inclusion complète de l'intervenant dans la problématique nécessitant un changement.

\section{Une grille d'analyse : quatre « registres d'intervention »}

11 Le croisement de l'ensemble de ces repères présentent une approche assez complète de la notion d'intervention psychosociologique et conduit à identifier quatre registres d'intervention assez typiques.

\begin{tabular}{|c|c|c|c|}
\hline \multicolumn{2}{|c|}{} & \multicolumn{2}{c|}{$\begin{array}{c}\text { Mode d'intervention } \\
\text { (Rapport au changement) }\end{array}$} \\
\cline { 2 - 4 } & Distancié & Recherche & Formation \\
\hline $\begin{array}{c}\text { Type } \\
\text { d'implication }\end{array}$ & Impliqué & Audit & $\begin{array}{c}\text { Intervention } \\
\text { (Approche } \\
\text { clinique) }\end{array}$ \\
\hline $\begin{array}{c}\text { (Rapport au } \\
\text { système client) }\end{array}$ & & \begin{tabular}{c} 
Inductif \\
\hline
\end{tabular}
\end{tabular}

Tableau I : Intervention psychosociologique et induction de changement

\section{La recherche}

Le type d'intervention répondant le mieux au croisement du mode de changement investigatif et du type d'implication distancié est, à notre sens, la recherche. En type pur, aucun changement direct n'est visé par ce type d'intervention. Il s'agit de recherche, c'est-à-dire d'une production de connaissances nouvelles (qui pourront éventuellement conduire à gérer le changement).

Un bon exemple de ce genre de travail est sans doute ce qu'on appelle aujourd'hui, à la suite des travaux d'A. Touraine, de F. Dubet ou de L. Van Campenhoudt, par exemple, «l'intervention sociologique «Lorsque F. Dubet (1987) réunit des groupes de jeunes banlieusards en France et en Belgique, son problème n'est pas de modifier les conditions d'existence des jeunes des grandes zones urbaines, mais de mieux comprendre les phénomènes sociaux qu'ils y vivent. Il s'agit de produire une meilleure intelligibilité de ce qui fait la vie quotidienne de ces jeunes. Et lorsque R. Quivy, D. Ruquoy et L. Van Campenhoudt (1989) travaillent avec des groupes d'enseignants au sein d'une école, le but n'est pas de changer cette école, de mettre fin à son «malaise ", mais bien d'essayer de 
mieux comprendre ce qui s'y passe et de tenter de décrypter ce qui fonde la dynamique sociale de cette école, dont certains acteurs peuvent se plaindre par ailleurs.

Mais dans ces deux exemples, la terminologie d'intervention sociologique est utilisée dans la mesure où la méthode mise en œuvre exige que les acteurs concernés (les jeunes, les enseignants) soient rencontrés sur leur terrain et qu'ils participent effectivement à l'interprétation et à la validation des explications faites par le chercheur en acceptant aussi de prendre part à un groupe d'analyse pour le temps de la recherche. C'est sans doute en cela que cette méthode se distingue le plus nettement de la recherche participative (les chercheurs ne se font pas membres du groupe social qu'ils étudient, serait-ce même en y prenant un statut spécial, comme chez W. F. White (1943), par exemple) et de la recherche-action: il n'y a pas à la fois vérification d'hypothèses et induction de changement (comme chez K. Lewin (1947), par exemple).

\section{L'audit}

Le croisement du mode investigatif avec un type de relation impliqué donne un deuxième modèle d'intervention psychosociologique dont l'audit, l'étude et l'expertise sont sans doute de bons exemples types. Nous pensons plus particulièrement aux audits de diagnostic et aux études ou expertises portant sur les dimensions humaines et sociales des entreprises, des administrations ou des associations (analyse de dysfonctionnements dans la communication interne, mauvaise ambiance, climat détérioré, crise dans les relations de travail, besoins de formation, évaluation d'un programme de formation, étude des caractéristiques de la culture d'une entreprise ou de l'effet d'un projet d'entreprise, étude d'images, etc.).

Dans ce cadre, l'intervenant met généralement en œuvre les ressources des différentes méthodes de recherche en sciences sociales (entretiens individuels et de groupes, enquêtes, recherche documentaire, analyse(s) de contenu, observation, etc.) pour effectuer un recueil et un traitement méthodique d'informations à propos d'un «problème » pour l'exploration systématique duquel il a été mandaté. L'enjeu ici est de mettre en œuvre correctement et avec éthique (pensons à la manière dont les résultats sont ou non restitués aux acteurs, par exemple) les méthodes de recherche les plus appropriées (Quivy et Van Campenhoudt, 1988; Grawitz, 1993; Albarello, Digneffe, Maroy, Ruquoy et de Saint-Georges, 1995) et de produire en définitive sous une forme synthétique un ensemble d'informations et de réflexions devant permettre aux décideurs de mieux cerner une situation et donc d'agir en meilleure connaissance de cause. Au besoin, le référentiel lui-même est construit par l'auditeur.

17 Mais on notera que l'induction du changement ne relève pas dans ce contexte de la responsabilité des intervenants et que par rapport à la problématique de la recherche, un certain nombre de paramètres sont négociés ou dictés ici par le mandat et ne relèvent donc pas de la complète autonomie de l'intervenant comme c'était le cas dans le type précédent. C'est d'ailleurs ce qui caractérise le mode de relation impliqué dans lequel se situent ces audits (d'où l'importance de la contractualisation des services attendus).

\section{La formation}

Venons-en maintenant à l'articulation de l'axe « mode inductif de changement » avec les types distanciés et impliqués de relation entre le système intervenant et le système client. 
19 La formation, sous l'angle des politiques et des pratiques de formation au sein des organisations, nous paraît bien caractériser l'articulation d'une visée inductive de changement avec un type de relation relativement distancié entre l'intervenant (interne ou externe) et son client.

20 Concevoir et mettre en œuvre une politique de formation vise généralement bien à induire du changement dans l'organisation. Qu'il s'agisse de changements liés à l'activité de travail sur un plan technique spécifique à un métier (par exemple introduire de nouvelles machines sans rupture de la production ou apporter des modifications dans les façons d'opérer suite à l'informatisation d'une procédure), qu'il s'agisse d'encourager certains comportements (par exemple : l'accueil de la clientèle, la manière de diriger le personnel ou celle d'animer un cercle de qualité, la façon de mener un entretien d'évaluation, la préparation d'une interview télévisée, etc.) ou encore qu'il s'agisse de vouloir améliorer le fonctionnement global d'un service ou de l'organisation (par exemple : organiser un séminaire de formation à la communication pour l'ensemble des ingénieurs d'une entreprise fusionnée, préparer la mise en place d'un projet ISO 9000, etc.).

21 Ces changements se mesurent d'ordinaire (lorsqu'ils se mesurent, bien entendu !) à la fois en termes individuels (apprendre est en définitive une affaire personnelle pour chacun d'entre nous), mais aussi au plan organisationnel: la mise en œuvre d'un plan de formation conduit normalement à des résultats voulus et perceptibles dans l'organisation.

22 Mais sur ce dernier point, il nous semble utile de souligner au passage deux choses essentielles : d'abord, rappeler la nécessité de mesurer l'impact d'une formation à partir de critères s'appliquant à un processus de formation. C'est une erreur assez fréquente, par exemple (a contrario), d'évaluer la qualité d'une formation en termes de personnes retrouvant un emploi à son issue, car il y a changement de niveau logique dans ce « saut " évaluatif. Sans doute la formation aide-t-elle à trouver un emploi, mais elle ne le crée pas; l'indicateur de mesure "personnes (re)trouvant un emploi » n'est par conséquent pas pertinent pour apprécier la qualité de la formation acquise ou donnée. Par contre, on n'a mesuré dans ce cas de figure, ni les méthodes, ni les apprentissages réalisés et on ne pourra donc pas réguler le dispositif de formation en y incluant ces données pourtant essentielles.

En second lieu, l'évaluation de l'impact d'un programme de formation sur l'organisation elle-même n'est pas nécessairement prise en charge par les personnes ou le service responsable de la formation. D'autres personnes ou services interviennent la plupart du temps dans cette procédure. Ceci permet de souligner l'importance extrême de l'articulation de ces services entre eux à la fois dans la conception, dans la mise en œuvre et dans l'évaluation de la formation pour éviter après coup (et coûts!) des malentendus largement basés sur des attentes mythiques ou magiques des uns et des autres vis à vis de la formation, voire des règlements de comptes entre services allant parfois jusqu'à mettre des intervenants extérieurs dans une position de «fusibles » assez caractéristique.

La gestion du changement organisationnel par la formation passe à notre avis par l'indispensable articulation de ces deux plans (individuel et organisationnel), par le contrôle des variables d'évaluation des résultats à leur sujet et par la mise en place d'un dispositif de pilotage le plus fin possible de l'intervention elle-même. 

système intervenant et le système client dans un contexte de formation: l'intervention consiste à conduire un projet de formation pour le mener à bien. Parfois, elle démarre avec l'étude préalable; dans d'autre cas, elle commence avec la conception du dispositif de formation, du programme et du choix des formateurs ; dans d'autres cas encore, par la mise en œuvre du programme de formation proprement dit et enfin elle peut ou non inclure la responsabilité d'évaluer les acquisitions au niveau des participants comme au niveau des retombées organisationnelles.

\section{L'intervention clinique}

Reste à conjuguer un mandat d'induction de changement avec un type impliqué de relation au sein de l'intervention. C'est ce que nous qualifions de travail, d'approche ou d' intervention clinique.

Dans ce cas de figure, il y a le plus souvent problème, voire même crise, c'est-à-dire perturbation plus ou moins importante de tout ou partie des modalités d'équilibration du système de personnes en interaction que constitue l'entreprise, l'administration ou l'association considérée. Et la mission d'intervention consiste à résoudre ce problème sans le déplacer ailleurs dans l'organisation. Selon les cas, la direction et les personnes impliquées ou leurs collègues ont une conscience plus ou moins nette de ce qui se passe, au moins au point de justifier la décision de recourir à un type de professionnels particuliers, capables en principe de prendre les choses en charge.

Il s'agit là d'un travail "clinique » dans la mesure où il faut chaque fois identifier dans l'organisation le niveau d'analyse paraissant le plus approprié compte tenu des difficultés mentionnées et sans négliger le contexte social, découvrir les symptômes de dysfonctionnement, porter un diagnostic pertinent en ce compris sur ce qui a pu faire échouer les initiatives de changement antérieures généralement présentes, déterminer des objectifs de changement aussi précis et réalistes que possible, puis conduire la stratégie de changement dans la durée en imaginant les modalités de "traitement " adéquates, le tout, la plupart du temps, en collaboration avec l'ensemble ou une partie des acteurs concernés et en veillant à conserver des liaisons positives de changement avec les instances de décision qui elles-mêmes peuvent parfois se trouver en déséquilibre plus ou moins important...

\section{Deux exemples de recours symptomatiques à la formation}

L'examen de la notion d'intervention psychosociologique nous a permis d'établir quatre grands registres d'intervention dont on peut généralement distinguer assez nettement les indications mais qui parfois s'interpénètrent et se confondent sur le terrain. Les distinctions apportées, même si la pratique conserve souvent la seule dénomination générique d'intervention (ou parfois de "formation») pour qualifier l'ensemble des quatre registres, se révèlent à l'expérience très utiles à la fois pour les décideurs des organisations et pour les intervenants eux-mêmes. Elles aident en effet à cerner avec plus de précision la nature du mandat d'intervention à préférer dans la situation 
problématique vécue et par conséquent à mieux se donner les moyens de gérer le changement. cohérence méthodologique propre: la recherche, l'audit, la formation et l'intervention clinique. Et dans la perspective de la gestion du changement qui nous occupe ici, chaque intervenant va normalement rechercher un contrôle aussi rigoureux que possible de sa méthodologie de travail, sous peine de se révéler très vite un piètre professionnel. Il a donc le choix entre quatre stratégies principales d'intervention.

31 Mais il est important de noter que ce «jeu de marelle» dans le concept et la méthodologie d'intervention ne permet pas en réalité de passer indifféremment d'une case à l'autre. Et dans certains cas, l'entrée par une mauvaise "porte» peut même conduire à une impasse, c'est-à-dire à la constatation qu'il aurait été méthodologiquement nécessaire de poser le problème autrement, mais à un moment où il n'est souvent plus possible de sortir de l'option qui a été prise.

Or à ce sujet, il nous semble, d'après notre expérience et nos recherches, que ce risque est le plus grand lorsque la demande d'intervention initiale est formulée par l'organisation en termes de formation. Bien sûr toutes les demandes de formation ne doivent pas pour autant être systématiquement mises en doute, mais tout se passe néanmoins parfois comme si certains problèmes vécus dans l'entreprise, l'administration ou l'association et réclamant un changement ne pouvaient être énoncés par l'organisation que comme des problèmes de formation.

cela qui va nous intéresser maintenant, car il s'avère la plupart du temps très difficile de passer en cours de route et sans rupture, d'une logique de formation à une logique de recherche, d'audit ou d'intervention alors que, par contre, l'inverse ne pose habituellement pas de problème majeur. Cette situation est assez inconfortable pour l'organisation comme pour l'intervenant lorsque le processus est mal engagé ; elle est cependant tout à fait intéressante pour réfléchir aux méthodologies d'induction de changement et à la place qu'y occupe parfois la formation comme symptôme exprimable face à des problèmes de changements organisationnels qui pour toutes sortes de raisons le sont nettement moins bien. Illustrons ceci par la présentation de deux exemples concrets.

\section{La formation à l'accueil}

Discutons un premier exemple. Il s'agit d'un programme de formation à l'accueil destiné au personnel ayant à exercer des fonctions de contact avec les patients d'un hôpital. Le programme a été mis en place suite à différents audits qui ont débouché sur l'élaboration par la direction d'un projet de "qualité totale » des services au sein de l'institution. Le projet général est géré par la direction qui en a confié la responsabilité opérationnelle à un « responsable qualité « Les formations quant à elles sont confiées depuis longtemps au service formation qui, selon les cas, les assure lui-même ou les sous-traite à des personnes ou à des organismes extérieurs après appel d'offre et sélection.

Le programme dont il est question ici s'inscrivait dans le cadre du "projet qualité » Il comportait trois journées par groupe d'une douzaine de personnes. Conformément au cahier des charges de l'appel d'offre, le programme et le déroulement des journées avaient été approuvés par les responsables de la formation. Ils étaient standardisés de 
manière à ce que les participants puissent avoir le sentiment qu'ils avaient tous vécus la même formation quel que soit le formateur. Plusieurs formateurs - tous professionnels et ayant de l'expérience - étaient en effet chargés d'animer chacun un ou plusieurs cycles de trois jours.

Le scénario de formation retenu prévoyait que les participants travaillent par des méthodes actives les situations d'accueil qu'ils vivaient eux-mêmes afin d'y relever les difficultés rencontrées et de déterminer des objectifs d'amélioration en termes de comportements personnels. La dimension organisationnelle de l'accueil était expressément écartée de la formation; le cas échéant, il était prévu à ce sujet de demander au groupe de garder la trace de ses réflexions, mais de rester centré avant tout sur les comportements.

co formation est mis en route sur cette base faisant l'objet d'un accord forme entre le responsable de la qualité, le responsable du service de formation et le responsable des formateurs extérieurs, soit les principaux interlocuteurs ayant finalisé le programme.

première journée du premier groupe se termine en respectant globalement le découpage des séances tel qu'il avait été planifié, mais en ayant consacré nettement plus de temps que prévu a priori aux réflexions concernant les aspects organisationnels de l'accueil, sur lesquels les participants s'étaient assez bien étendus. Du point de vue du formateur, les choses ne s'étaient pas trop mal passées pour un début, la participation avait été active et intéressée et il restait encore deux journées pour aboutir aux objectifs. Mais dès la fin de cette première journée du premier groupe cependant, c'est l'effervescence au sein du service formation de l'hôpital. Le responsable de ce service interpelle le soir même par téléphone le coordinateur (extérieur) des formateurs du programme : «la journée de formation s'était vraiment très mal passée », le programme prévu n'avait pas été respecté, on n'avait fait que critiquer l'organisation des services et le formateur n'avait pas été capable d'y mettre fin », etc. Le tout était asséné avec grande émotion mais sans s'appuyer sur aucune procédure d'évaluation formelle et en mettant expressément en cause les capacités professionnelles du formateur dont on demandait à ce qu'il soit séance tenante retiré du circuit. Que s'était-il donc passé pour qu'il y ait si vite une réaction aussi vive du responsable de la formation?

Les choses s'étant quelque peu calmées et les contraintes concrètes ayant inspiré un certain réalisme, au moins provisoire, (on ne remplace pas comme cela un formateur en plein travail), la formation s'est poursuivie et les participants ne s'en sont pas du tout montrés mécontents si on en juge cette fois par ce qui a été exprimé formellement lors de l'évaluation faite à l'issue du cycle complet. Mais plusieurs d'entre eux soulignaient à cette occasion qu'ils avaient déjà participé à une formation à l'accueil de ce genre il y a quelque temps, que des formations comme cela étaient intéressantes, mais qu'elles ne changeraient rien parce que le problème n'était pas là.

41 En réalité disaient la plupart des participants, « la direction n'ose pas traiter certaines choses » alors que cela pourrait apporter une solution plus directe à la qualité de l'accueil et plus généralement de leur travail. A leur avis, par exemple, même parfaitement formés à l'accueil, il serait toujours difficile pour eux de sourire et de faire patienter pendant un temps assez long les personnes arrivées à l'ouverture du service alors que l'on sait très bien que le médecin effectuant les consultations dans ce service n'arrivera que nettement plus tard, comme d'habitude. "S'il y avait moyen de le lui dire et surtout de faire en sorte qu'il change cette attitude, ne serait-ce qu'un peu » (même si elle pouvait avoir des 
fondements inconnus des participants), alors « accueillir les patients serait facile et on verrait vite ceux et celles qui auraient encore vraiment besoin de formation à ce sujet... »

Des propos de ce genre avaient été tenus par les participants dès le premier jour; ils avaient été enregistrés par le formateur suivant les consignes de départ pour en garder la mémoire; Mais du point de vue du service formation, ces considérations n'étaient pas dans le champ du séminaire (il devait porter sur les comportements individuels, rappelons-le) et à y regarder de plus près, les considérations des participants pouvaient apparaître comme une menace ou un danger. Dans la mesure où elles étaient pertinentes, elles mettaient en effet directement en cause l'option de s'en tenir aux comportements d'accueil pour atteindre la qualité de service recherchée et donc la logique même du cahier des charges (d'où sans doute la promptitude et la vigueur de la réaction du service formation).

Mais par ailleurs, pour la direction et le responsable de la qualité, la formation mise en place devait évidemment contribuer comme telle à l'amélioration de l'accueil des patients dans le cadre général du " plan qualité ». L'enquête auprès des patients n'avait-elle pas montré que l'accueil par le personnel était souvent considéré comme déficient?

On voit le malentendu. En outre, face au risque d'un changement organisationnel pouvant toucher à certaines catégories plus «sensibles" (comme les médecins, par exemple), chacun se devait sans doute aussi d'être prudent, consciemment ou non. La direction générale avait fait effectuer une analyse des besoins de formation ; le service qualité avait promu la nécessaire formation à l'accueil ; le service formation l'avait mise sur pieds ; les membres du personnel y participaient. Quoi d'étonnant à ce qu'ils tiennent à faire savoir que s'il y avait un problème d'accueil, il ne fallait pas déduire de leur participation à un séminaire qu'ils en étaient la cause ? Et le formateur servait sans doute un peu de fusible à tous.

\section{La formation à la communication pour les ingénieurs}

Prenons un autre exemple. La direction d'une entreprise industrielle demande que nous animions un séminaire de formation à la communication pour ses ingénieurs. Nous convenons donc d'avoir un entretien préalable afin d'explorer la question de manière plus complète avant de faire une proposition de séminaire de formation répondant au mieux aux problèmes qui se posent. La rencontre se passe dans l'entreprise. Elle nous met en présence, un collègue et moi-même, avec la direction du siège, demandeur de la formation, et le directeur du personnel «Belgique « de cette entreprise multinationale. Momentanément en effet, nous dit-on, il n'y a plus pour l'instant de directeur du personnel dans ce siège.

L'entretien porte comme prévu sur la demande d'organisation d'une formation à la communication pour la douzaine d'ingénieurs travaillant dans cette usine. L'entretien fait apparaître qu'il y a en réalité deux sous-ensembles parmi les ingénieurs : les uns sont les ingénieurs du site et les autres font partie de la cellule de recherche "Europe «de l'entreprise. Ces derniers font cependant leurs essais sur les équipements du siège, ce qui cause parfois certaines perturbations dans les lignes de production. Ils sont dirigés par un directeur de la recherche «Europe «qui a ses bureaux sur le site, contigus à ceux du directeur du siège. Ce directeur de la recherche ne pouvait pas être présent à la réunion. 
47 Arrêtons-nous un moment dans cette description pour noter qu'une réponse immédiate aurait pu être donnée dès les premiers contacts téléphoniques. Elle aurait probablement eu pour effet de mettre sur pieds un programme de formation " prêt à porter » comme on en trouve pas mal dans les brochures de différents organismes de formation. La demande explicite était bien d'organiser une telle formation et elle faisait suite à une analyse interne des initiatives à prendre pour que certaines choses changent dans l'entreprise. Il faut souligner aussi que nous ne mettions pas en doute le bien fondé de la demande de la direction, mais plutôt que d'offrir un produit standard, nous voulions aller plus loin dans l'étude de la commande qui nous était faite afin de profiler notre proposition au mieux des intérêts de l'entreprise et de nos compétences. Cela ne se règle pas en deux ou trois coups de fils entre inconnus.

En revenant à l'entretien initial, on observera maintenant que les interactions entre les deux sous-ensembles d'ingénieurs, contrairement à ce qu'on pourrait croire à première vue, ne faisaient généralement pas problème aux yeux de nos interlocuteurs. Autrement dit, l'hypothèse selon laquelle une formation à la communication aurait pu être motivée par une difficulté de contact entre les ingénieurs sur base des deux sous ensembles qu'ils formaient dans l'entreprise (production recherche) ne se révélait pas féconde. Pourtant il fallait former les ingénieurs à la communication...

49 Par contre, l'existence d'un autre directeur (recherche « Europe»), absent à l'entretien, et l'absence momentanée de directeur du personnel du siège ouvraient des pistes de réflexions toutes autres, mais cette fois au niveau même du management. Rien ne nous autorisait cependant à déplacer le problème d'une formation à la communication pour ingénieurs vers ce genre d'hypothèses. Mais la méthodologie adoptée consistait à mener un entretien systématique, auquel nos interlocuteurs se prêtaient d'ailleurs de bonne grâce, et donc à prendre une position momentanée d'intervenant clinique cherchant à diagnostiquer la situation avant de proposer un programme de travail.

La suite de l'entretien fit apparaître d'autres informations. Tout d'abord, il sembla vite assez clair que l'absence d'un responsable du personnel pouvait sans doute être considérée comme fortuite et momentanée. Par contre, les interactions au sein de la direction devaient être investiguées plus avant. Et il apparut que le directeur du siège et le directeur de la recherche avaient été alternativement sous les ordres l'un de l'autre dans d'autres fonctions et dans d'autres sièges de l'entreprise avant de connaître la situation actuelle. Celle-ci pouvait être décrite comme deux fonctions de direction à la fois indépendantes et tributaires l'une de l'autre. Hiérarchiquement, les deux directeurs ne relevaient pas l'un de l'autre, mais opérationnellement leurs troupes (les ingénieurs qui avaient besoin d'une formation à la communication) se trouvaient fréquemment en train de dépendre et de l'un et de l'autre pour faire au quotidien ce qu'ils avaient à faire.

51 À l'issue de cette rencontre exploratoire, il a été convenu que la proposition de formation serait élaborée en deux temps : une phase préalable d'interviews des ingénieurs et des deux directions afin de repérer les problèmes de communication spécifiquement vécus par ces acteurs; ensuite, sur cette base, il y aurait conception et proposition d'un séminaire de formation «sur mesure ». L'interview des deux directeurs sur cette même question paraissait essentielle à tous les interlocuteurs présents pour pouvoir faire un travail pertinent dans le contexte du siège. 
était posée comme devant relever de toute évidence d'une initiative de formation. Dans le second exemple comme dans le premier, le recours à la formation a ainsi fonctionné non pas comme un projet de changement organisationnel par la formation, mais bien plus comme un symptôme, comme une manière acceptable de pouvoir exprimer un dysfonctionnement dans le système organisationnel.

\section{La problématique de l'intervention : changer de changement}

57 Le cadre théorique relatif à l'induction de changement permettant de discuter plus complètement les observations que nous venons de faire est familier aux psychothérapeutes et aux psychosociologues se référant aux théories pragmatiques et systémiques de «la nouvelle communication »(Winkin, 1981). Il l'est sans doute moins pour les intervenants se réclamant d'autres cadres de référence et moins encore, et c'est évidemment bien compréhensible, pour les responsables d'organisation ayant à gérer des problématiques de changements. Nous allons donc en donner un bref aperçu et conclure notre analyse. (Voir aussi les orientations bibliographiques en annexe). 

l'administration) est considérée comme un système de communication, c'est-à-dire comme un ensemble de comportements en interaction (Selvini Palazzoli et al., 1984). Concevoir tout comportement comme un acte de communication est l'élément de base de la pragmatique de la communication. Elle en tire d'ailleurs sa dénomination (pragmatique). Ces comportements, ces interactions, s'inscrivent dans une hiérarchie de contextes qui peuvent délimiter autant de niveaux d'analyse ou d'intervention (par exemple : le niveau des relations interpersonnelles, le niveau du groupe restreint, celui d'une organisation ou d'un de ses services, le niveau des grands ensembles sociaux (institutions, milieux), celui d'une société). couple et ses enfants; les parents du couple, le couple et ses enfants; les voisins de palier et cette famille?), ni en ce qui concerne une organisation (est-ce la direction, un service, une catégorie de membres du personnel, l'ensemble de l'entreprise ?). C'est en fonction de quelque chose (plainte, problème, demande, hypothèse de travail, etc.) que quelqu'un (thérapeute, intervenant, chercheur) détermine en définitive le système qu'il espère pertinent en regard de ce fil conducteur.

Tout système ainsi délimité va, par définition, tendre à conserver son équilibre, à maintenir sa stabilité, son homéostasie. Autrement dit, face aux perturbations qui affectent inévitablement le fonctionnement concret et quotidien du système d'interaction, les membres de ce système vont prendre des initiatives pour restaurer l'équilibre menacé ou perdu en communiquant, en s'activant dans le cadre (et parfois en dehors) des règles du jeu qui régissent leurs interactions. L'équilibration du système, la possibilité dynamique de poursuivre le jeu, ne doit donc pas être confondue avec une invariance conservatrice. Du point de vue développé ici, maintenir la stabilité du système est déjà un phénomène de changement dans la mesure où cela demande de prendre chaque jour des initiatives de gestion des perturbations (décider, se parler, se tirer ou non la tête, organiser une réunion, engager quelqu'un, «mettre les choses au point ", entrer en conflit, former, négocier, effectuer un audit, fusionner ou créer de nouveau services, informer, participer ou non, aménager l'espace et le temps, etc.).

61 Il arrive toutefois que certaines perturbations persistent malgré les initiatives de changement prises par les membres du système. Dans ce cas, la façon habituelle de réguler les difficultés ( les règles du jeu », la «culture de l'entreprise ») ne permet plus de trouver une solution. De difficultés adaptatives, le système en vient à une situation où les efforts réalisés pour restaurer la possibilité de poursuivre ensemble le jeu communicationnel (ou si on veut, organisationnel) ne ramène plus l'équilibre du système d'interaction. Il y a problème, voire crise des modalités de régulation elles-mêmes et le système peut jusqu'à se trouver menacé d'existence.

Dans cette hypothèse, un autre type de changement devient nécessaire. On peut le décrire comme visant non plus à changer le jeu entre acteurs, mais bien à changer les règles du jeu pour réussir à résoudre les problèmes rencontrés. Bien entendu, les personnes concernées n'ont pas nécessairement une vue claire des possibilités offertes par cette stratégie éventuelle de changement, même si elles peuvent avoir une perception aiguë des problèmes qu'elles vivent entre elles et la plupart du temps, pour certaines au moins, en souffrir. 
63 dans la voie qui leur est habituelle et ils y témoignent parfois d'une très grande créativité. Ils se trouvent ainsi dans la situation que Watzlawick et al. (1975) décrivent comme un «jeu sans fin » Il consiste en définitive à ce que pas mal de gens consacrent beaucoup d'énergie à changer les choses sans arriver à le faire (" plus ça change, plus c'est la même chose »). L'enjeu de la crise est alors de parvenir à «changer de changement "pour produire une modification « systémique »et en principe résoudre les problèmes.

L'intervention psychosociologique apparaît dans ce contexte. Elle est donc une perturbation délibérée d'un système de communication (ensemble contextualisé de comportements en interaction) effectuée par des professionnels à un ou plusieurs niveaux d'analyse, en vue d'induire un changement devenu nécessaire par l'existence de problèmes persistants que les membres du système n'arrivent plus à réguler malgré des efforts dans ce sens. Et les quatre registres que nous avons proposés en sont des modulations stratégiques particulières.

Quoique présenté ici très brièvement, ce cadre de référence permet d'expliquer en profondeur comment les initiatives de formation décrites dans les exemples abordés peuvent à la fois être réellement et pleinement destinées à changer les choses et cependant ne pas conduire au changement organisationnel souhaité. Il permet surtout de comprendre pourquoi, dans une situation donnée, on ne peut méthodologiquement pas conduire l'induction du changement en entrant par n'importe quelle case du « jeu de la marelle " proposé par notre schéma: on aura noté que l'entrée dans la conduite du changement par la formation est définie dans ces termes par l'organisation, mais elle n'est méthodologiquement pas nécessairement construite pour autant par l'organisation comme une intervention, au sens précis du terme en matière de conduite du changement. Voilà sans doute pourquoi, sous peine d'accepter a priori d'entrer dans un «jeu sans fin ", il sera chaque fois méthodologiquement nécessaire de construire le dispositif d'intervention en déterminant et le type de relation et le mode d'intervention appropriés.

Dans le premier exemple, chacun faisait sans doute son travail du mieux qu'il pouvait, mais au total l'effort de changement restait systématiquement improductif, sinon, comme cela aurait pu arriver aussi dans un tel contexte, contre-productif. Il pouvait être dit qu'il $\mathrm{y}$ avait un problème d'accueil et que les membres du personnel pouvaient et devaient améliorer leurs capacités personnelles en cette matière ; il n'était sans doute pas encore acceptable d'aller au-delà et de constater que des initiatives étaient aussi à prendre sur le plan organisationnel. Si on ne l'arrêtait pas tout de suite, le formateur prenant trop de temps avec les participants sur les aspects organisationnels du changement, risquait de mettre en cause le fragile équilibre dont tout le monde pouvait sans cela continuer de s'accommoder.

67 Le piège d'une double contrainte aux implications multiples s'est ainsi refermé à la fois sur les membres du personnel en formation (« soyez accueillant»), sur ceux qui conçoivent et assurent cette formation (" formez à l'accueil, gardez la mémoire des aspects organisationnels s'il y en a, mais ne les traitez pas ») et de fil en aiguille sur le service responsable de sa gestion voire en définitive sur le management («nous voulons la qualité totale et il y a un service pour cela ; ça va changer puisqu'on l'a créé »).

Dans le second exemple, le processus d'induction de changement a été méthodologiquement piloté très différemment par les acteurs. Au départ l'entreprise l'a posé aussi comme un projet de formation. Mais en acceptant d'aborder le problème avec 
les intervenants auxquels elle recourait, par l'entrée de l'« intervention clinique » plutôt que par l'entrée "formation ", l'espace de travail s'est trouvé suffisamment élargi pour pouvoir y introduire, sans exclure l'éventualité d'un projet de formation, une chance de changement auquel beaucoup aspiraient.

\section{BIBLIOGRAPHIE}

ALBARELLO L, DIGNEFFE F, HIEMAUX J.P, MAROY Chr, RUQUOY D. et DE SAINT-GEORGES P, Pratiques et méthodes de recherche en sciences sociales, Paris, Armand Colin, 1995.

AMBLARD A, BERNOUX Ph, HERREROS G, LIVIAN Y-F, Les nouvelles approches sociologiques de l'organisation, Paris, Seuil, 1996.

AUBERT N. et DE GAULEJAC V, Le coût de l'excellence, Paris, Seuil, 1991.

BERIOT D, Du microscope au macroscope : l'approche systémique du changement dans l'entreprise, Paris, ESF, 1992.

BONAMI M, DE HENIN B., BOQUE J.-M. et LEGRAND J.J, Management des systèmes complexes, Bruxelles, De Boeck, 1993.

BOTTIN Chr., Diagnostic et changement : l'intervention des consultants dans l'organisation, Paris, Ed d'organisation, 1991.

DEFOURNY V., Organisation et pilotage stratégique de la communication, Louvain-la-Neuve, Faculté des sciences économiques, sociales et politiques, 1991.

DE SAINT-GEORGES P., «Culture d'entreprise, communication interne et stratégies de changement », in Communication et organisation, 4, 1993.

DUBET F., La galère : jeunes en survie, Paris, Fayard, 1987.

DUBOST J., L'intervention psychosociologique, Paris, P.U.F, 1987.

DUTERME Cl., Organisation et changement : un regard sur l'intervention, Louvain-la Neuve, UCLFOPA, 1992.

EVEQUOZ G, Le contexte scolaire et ses otages, Paris, ESF, 1984.

FISCH R., WEAKLAND J.H. et SEGAL L, Tactiques du changement, Paris, Seuil, 1986.

FRIEDBERG E., Le pouvoir et la règle : dynamique de l'action organisée, Paris, Seuil, 1993.

FRIEDBERG E., « Les quatre dimensions de l'action organisée », in Revue française de sociologie, 33, 1992, pp. 532-557.

GRAWITZ M., « Recherche active et intervention psychosociologique », in Méthodes des sciences sociales, Paris, Dalloz, 1993.

HALEY J., Nouvelles stratégies en thérapie familiale, Paris, Delarge, 1979.

«L'audit de la formation », in Education Permanente, 91, 1988.

LAYOLE G., Dénouer les conflits professionnels, Paris, Les Éditions d'organisation, 1984. 
«Lectures systémiques des organisations » in Cahiers critiques de thérapie familiale et de pratiques de réseaux, n 13, 1991.

LEWIN K., « Décisions de groupe et changement social », in LEVY A., Psychologie sociale : textes fondamentaux anglais et américains, Paris, Dunod, 1972.

MINTZBERG H., Stucture et dynamique des organisations, Paris, Les Éditions d'organisation, 1982.

NARDONE G. et WATZLAWICK P., L'art du changement, Bordeaux, L'esprit du temps, 1993.

NIZET J. et PICHAULT F., Comprendre les organisations. Mintzberg à l'épreuve des faits, Paris, Gaëtan Morin, 1995.

PAGES M., «Les options fondamentales de l'intervention psychosociologique », in La vie affective des groupes, Paris, PUF, 1968.

PAGES M., «La pratique de l'intervention psychosociologique », in La vie affective des groupes, Paris, PUF, 1968.

PAGES M., BONETTI M., DE GAULEJAC V. et DESCENDRE D., L'emprise de l'organisation, Paris, PUF, 1992.

PETIT F., Introduction à la psychosociologie des organisations, Toulouse, Privat, 1984.

PIRSON J., « De l'analyse stratégique à l'analyse pragmatique », in Recherches sociologiques, 3, 1999, 131-146.

QUIVY R., RUQUOY D. et VAN CAMPENHOUDT L., Malaise à l'école : les difficultés de l'action collective, Bruxelles, Facultés universitaires Saint-Louis, 1989.

SAINSAULIEU R., Sociologie de l'organisation et de l'entreprise, Paris, Dalloz, 1987.

SELVINI PALAZZOLI M., ANOLLI L, DI BLASIO P., GIOSSI L., PISANO I., RICCI C., SACCHI M. et UGAZIO V., Dans les coulisses de l'organisation, Paris, ESF, 1984.

SERON Cl. et WITTEZAELE J.J, Aide ou contrôle : l'intervention thérapeutique sous contrainte, Bruxelles, De Boeck, 1991.

WATZLAWICK P., HELMICK-BEAVIN J. et JACKSON D., Une logique de la communication, Paris, Seuil, 1975.

WATZLAWICK P., WEAKLAND J. et FISCH R., Changements, paradoxes et psychothérapie, Paris, Seuil, 1975.

WHYTE W.F..., Street corner society : the social structure of an Italian slum, Chicago : Univ. of Chicago Press, 1943, (trad. française, Paris, Éditions la découverte, 1996).

WINKIN Y., Anthropologie de la communication : de la théorie au terrain, Bruxelles, De Boeck, 1996.

\section{RÉSUMÉS}

L'organisation est un système de communication, un ensemble contextualisé de comportements en interactions. Souvent, des changements s'y produisent en dehors d'une quelconque politique : les membres de l'organisation s'adaptent et innovent. Dans d'autres cas, le management induira le changement face à des problèmes spécifiques. Mettre sur pied un programme de formation est une des réponses possibles. Mais il arrive que l'exigence de formation soit une manière acceptable d'exprimer que des changements d'une autre nature seraient nécessaires. Le recours à la formation prend valeur de symptôme sans qu'il soit certain qu'une telle initiative soit à 
conseiller pour atteindre les changements escomptés. Le texte situe la stratégie de formation parmi quatre modes d'induction du changement et montre combien il est préférable d'aborder l'intervention dans une posture initiale de recherche ou d'approche clinique.

The organization is conceived as a System of communication, a contextualized set of comportments in interaction. Changes occur spontaneously in organizations, independently of any systematic attempt to generale them. Members of the organization adapt to these changes and innovate. Management might also take on to induce change, to respond to some organizational issues. In these cases, training is one possible response to some punctual or recurring difficulty. The need for training may be just a way to express in acceptable terms a need for change of another nature. Training takes on the value of a symptom, when changes of some other kind would be necessary. In these cases, it is doubtful that training should be advised as a mode of action to produce the changes aimed for.

\section{AUTEUR \\ PIERRE DE SAINT-GEORGES}

Docteur en sociologie, Pierre de Saint-Georges est professeur à l'Université Catholique de Louvain et aux Facultés Universitaires Saint-Louis (Bruxelles). Ses recherches ont pour cadre théorique la pragmatique de la communication et sont centrées sur la problématique du changement et de l'induction du changement dans les groupes et les organisations. Il allie recherche et enseignement avec une expérience de conseil et d'intervention. Il préside le Centre d'Études de la Communication (CECOM) et est membre du Laboratoire d'analyse des systèmes de communication des organisations (LASCO) à l'UCL. 